\title{
A Hole Theory of Polymer Liquids and Glasses. II. Glass Transition and Glassy State; Equation of State
}

\author{
Takuhei Nose \\ Department of Polymer Technology, Tokyo Institute of \\ Technology, Tokyo, Japan.
}

(Received June 19, 1970)

\begin{abstract}
A hole theory of polymer glasses is presented, in which the glassy state is distinguished from the liquid state by the freezing of holes. The equation of state, internal pressure, and cohesive energy density are derived. The results of the theory are compared with experimental observations with the use of the molecular parameters obtained in the preceding paper. The hole theory can semiquantitatively explain the decrease of internal pressure on glass formation.

The free volume at the glass transition temperature and the correlation of the glass transition temperature to cohesive energy density are also discussed.

KEY WORDS Hole Theory/Polymer Glass/Glass Transition/Equation of State/Internal Pressure / Free Volume/Cohesive Energy Density/
\end{abstract}

In a previous paper $^{1}$ of this series we applied the hole theory to polymeric systems along the line of the theories of polymer liquids developed recently, ${ }^{2,3}$ and calculated the partition function and the equation of state in the liquid state. Here we will discuss the glassy state by the same approach.

The glassy state is, as is well known, a thermodynamically non-equilibrium state and depends on the condition of glass formation. Once the glass is formed, however, the relaxation associated with the microbrownian motion, e.g., volume relaxation, is not observed in the usual time scale of measurement at temperatures well below the glass transition temperature. Thus we here deal with the glassy state as a quasiequilibrium state, i.e., we regard it as a frozen liquid such that polymer chains cannot change their conformations and cannot exchange their mean positions with each other. In the glassy state the segments can only vibrate in the vicinity of their mean positions as in the crystalline state whereas the long range order of the configuration of molecules in the system as is found in the crystalline state does not exist. These features of the glassy state are characteristically manifested in the behavior of the "excess entropy" which is here defined as the entropy in excess of that of the perfect crystal at the same temperature. The excess entropy decreases with decreasing temperature till the glass transition temperature is reached, and remains almost constant below the transition temperature. The same behavior is also found in the case of "excess enthalpy". These phenomena may imply that the glassy state is very similar to the crystalline state in respect to temperatureand volume-dependences of themodynamic behavior. Corresponding to the above facts, the discontinuous decreases in internal pressure and heat capacity on glass formation are also observed, which reflects the change in the state of aggregation of molecules from the liquid-like state to the solid-like one. The excess entropy, however, is not zero in the glassy state. This may indicate that the glass has a configurational disorder which is different from the crystal.

It seems to the author that the theories developed recently for polymer liquids ${ }^{2,3}$ are unsuitable for extension to explain such differences of the glass from the liquid and the crystal, whereas a hole theory may explain them as the freezing of holes as far as the intersegmental interactions are concerned. ${ }^{1}$

In this paper we propose a theory of polymer glasses based on the above considerations, which is an extension of the hole theory for polymer liquids. ${ }^{1}$ We derive the equation of state, 


\section{T. Nose}

cohesive energy density, and internal pressure in the glassay state, and discuss the change in internal pressure at the glass transition temperature and the free volume at $T_{\mathrm{g}}$.

\section{THEORY}

\section{Partition Function}

We now consider a system in which $N / r$ polymer molecules, each consisting of $r$ segments, are arranged on a lattice comprising $M$ sites $(M \geq N)$, and therefore there remain $M-N$ empty sites or holes. According to Prigogine, ${ }^{2}$ we assume that the external degrees of freedom attributable to a segment are reduced to $3 c(c \leq 1)$ because of interconnection of segments, and that the coordination number $z^{\prime}$ of a segment becomes smaller than that of the lattice $z$ because a part of intermolecular contacts is replaced by intramolecular ones. $z^{\prime}$ is related to $z$ by the equation $z^{\prime}=(z r-2 r+2) / r$. Then the partition function $Z$ of the system may be written ${ }^{1}$

$$
\begin{aligned}
Z= & J(T) q^{c N} v_{\mathrm{f} 0}^{c N} \exp \left\{-z^{\prime} \varphi(0) N / 2 k T\right\} \\
& \times \sum_{\lambda} g_{\lambda} \cdot\left(v_{\mathrm{f} 1} / v_{\mathrm{f} 0}\right)^{c N(\widetilde{V}-1) /(\widetilde{V}-1+s)} \\
& \times \exp \left\{\frac{N \varphi(0) z^{\prime}(\tilde{V}-1)}{2 k T(\tilde{V}-1+s)}\right\}
\end{aligned}
$$

with

$$
\begin{aligned}
& \sum_{\lambda} g_{\lambda} \\
& =\{(z-1) / e\}^{(r-1) N / r} r^{N / r} \tilde{V}^{N(\tilde{V}-1)+N / r}(\tilde{V}-1)^{-N(\widetilde{V}-1)} \\
& v_{\mathrm{f} 1}=v^{*}=V^{*} / N
\end{aligned}
$$

and

$$
\varphi(0)=\varepsilon^{*}\left\{1.0109\left(\sigma^{3} N / V^{*}\right)^{4}-2.4090\left(\sigma^{3} N / V^{*}\right)^{2}\right\}
$$

where $J(T)$ is the partition function for the intrasegmental degrees of freedom, and $q^{c N}$ is the kinetic part of the partition function associated with the external degrees of freedom. The quantity $\sum_{\lambda} g_{\lambda}$ represents the number of configurations of molecules arranged on the lattice and we here used the result of Flory's theory ${ }^{4}$ for polymer solutions. The free volume $v_{\mathrm{f} 0}$ and the intersegmental interaction energy $\varphi(0)$ were evaluated by using the square well potential and the Lennard-Jones $12-6$ potential respectively, and $\varepsilon^{*}$ and $\sigma$ are the characteristic pa- rameters involved in the latter potential expressed as

$$
u\left(r_{i j}\right)=4 \varepsilon^{*}\left\{\left(\sigma / r_{i j}\right)^{12}-\left(\sigma / r_{i j}\right)^{6}\right\}
$$

where $u\left(r_{i j}\right)$ is the potential energy of interaction between molecules (or segments) $i$ and $j$ as a function of separation $r_{i j}, \varepsilon^{*}$ is potential energy at a minimum of $u\left(r_{i j}\right)$, and $\sigma$ is the separation $r_{i j}$ at $u\left(r_{i j}\right)=0$. The volume of a cell is denoted by $v^{*}$, which is defined by $v^{*}=V^{*} / N=V / M$. The quantities $\tilde{V}$ and $s$ are defined as $\tilde{V}=$ $V / V^{*}=M / N$ and $z^{\prime} / z$, respectively.

In the liquid state, both the number of holes $(M-N)[=N(\tilde{V}-1)]$ and the volume of the cell may change with volume and temperature, as treated in the previous paper. ${ }^{1}$. On the other hand, in the glassy state the number of holes is considered to be unchangeable because the rearrangement of segments on the lattice cannot be allowed. Thus we here assume that in the glassy state the number of holes $(M-N)$ remains constant. In other words, the values of $M / N$ $(=\tilde{V})$ is assumed to be constant and equal to $\widetilde{V}_{\mathrm{g}}=V_{\mathrm{g}} / V_{\mathrm{g}}{ }^{*}$, where $V_{\mathrm{g}}$ and $V_{\mathrm{g}}{ }^{*}$ are respectively $V$ and $V^{*}$ at the glass transition point. The change in volume in the glassy state, therefore, comes solely from a change in the cell volume. The hindered rotation about chain backbones which is related to $J(T)$ may also be frozen-in and not be allowed to change in the glassy state.

The external degrees of freedom $3 c$ of a segment are associated with the cell partition function and hence the free volume. Thus, since the effect of the interconnection of segments on the free volume, in other words, on the movement of the segment within the cell, may be considered to be the same in both the liquid and glassy states, the parameter $c$ in the glassy state is assumed here to be identical with that in the liquid state. In the present theory, the changeability of the chain conformations in the liquid state is expressed by the change in the configurations or the arrangements of polymer chains on the lattice.

Under this theory, therefore, the freezing of the microbrownian motion in the glassy state is represented by the freezing of both holes and the chain conformations.

Accordingly, using the partition function of 


\section{A Hole Theory of Polymer Liquids and Glasses}

eq 1 with eq $3-5$, under the condition $\tilde{V}=\tilde{V}_{\mathrm{g}}=$ constant, we can obtain the thermodynamic quantities in the glassy state which are associated with the intersegmental interactions and hence derivable from the volume-dependent part of $Z$. The hole fraction frozen-in in the glassy state, hence $V_{\mathrm{g}} / V_{\mathrm{g}}{ }^{*}$, is the so-called ordering parameter, and is a single variable which specifies the frozen structure of the glass concerned with the intersegmental state, excluding the intrasegmental state represented by $J(T)$. Thus the thermodynamic properties of the glass associated with the intersegmental interactions generally depend on $V_{\mathrm{g}} / V_{\mathrm{g}}{ }^{*}$ in addition to temperature and pressure. For example, the volume in the glassy state is not determined by temperature and pressure without specifying the third variable $V_{\mathrm{g}} / V_{\mathrm{g}}{ }^{*}$.

Equation of State, Cohesive Energy Density, and Internal Pressure

The equation of state can be derived from the partition function eq 1 with eq 3-5 under the condition, $\tilde{V}=\widetilde{V}_{\mathrm{g}}=$ constant, in the conventional manner:

$$
P=k T(\partial \ln Z / \partial V)_{\tilde{V}, T}
$$

The result is

$$
\begin{aligned}
& \tilde{P} \tilde{V}^{\prime}\left(\tilde{V}_{\mathrm{g}}-1+s\right) / s=\left(\tilde{V}_{\mathrm{g}}-1+s\right) / s \\
& \quad+\tilde{V}_{\mathrm{g}}^{1 / 3} 2^{-1 / 6} \tilde{V}^{\prime-1 / 3}\left(1-\tilde{V}_{\mathrm{g}}^{1 / 3} 2^{-1 / 6} \tilde{V}^{\prime-1 / 3}\right) \\
& \quad-\left(2.409 \tilde{V}_{\mathrm{g}}{ }^{2} \tilde{V}^{\prime-2}-2.0218 \tilde{V}_{\mathrm{g}}{ }^{4} \tilde{V}^{\prime-4}\right) / \widetilde{T}
\end{aligned}
$$

where $\widetilde{P}=P v^{*} / \varepsilon^{*} z^{\prime}, \tilde{T}=c k T / \varepsilon^{*} z^{\prime}$, and $\tilde{V}^{\prime}=V / \sigma^{3} N$.

By the use of eq 1 with eq 3-5 under the same condition, $\tilde{V}=\tilde{V}_{\mathrm{g}}=$ constant, as in the case of the equation of state, cohesive energy density $D_{\mathrm{g}}$ and internal pressure $\boldsymbol{P}_{\mathrm{ig}}$ in the glassy state are derived from

$$
D_{\mathrm{g}}=-\left(k T^{2} / V\right)\left(\partial \ln Z_{\mathrm{v}} / \partial T\right)_{V, \tilde{V}}
$$

and

$$
P_{\mathrm{ig}}=(\partial U / \partial V)_{T}=-\left[\partial\left(D_{\mathrm{g}} V\right) / \partial V\right]_{T, \tilde{V}}
$$

where $Z_{v}$ is the volume-dependent part of the partition function $Z$, i.e., $Z_{\nabla}=Z / J(T) q^{c N}$ in eq 1. Thus we have

$$
\begin{aligned}
D_{\mathrm{g}}= & \left(\varepsilon^{*} z^{\prime} / \sigma^{3}\right) s \tilde{V}_{\mathrm{g}}^{2}\left(\tilde{V}_{\mathrm{g}}-1+s\right)^{-1} \tilde{V}^{-3} \\
& \times\left(2.409-1.0109 \tilde{V}_{\mathrm{g}}^{2} \tilde{V}^{\prime-2}\right) / 2 \\
P_{\mathrm{ig}}= & \left(\varepsilon^{*} z^{\prime} / \sigma^{3}\right) s \tilde{V}_{\mathrm{g}}^{2}\left(\tilde{V}_{\mathrm{g}}-1+s\right)^{-1} \tilde{V}^{\prime-3} \\
& \times\left(2.409-2.0218 \tilde{V}_{\mathrm{g}}^{2} \tilde{V}^{\prime-2}\right)
\end{aligned}
$$

Change in Internal Pressure at Glass Transition Temperature

We will now consider the magnitude of the internal pressure difference between the glassy and liquid states at glass transition temperature. According to the results obtained in the previous paper, ${ }^{1}$ internal pressure in the liquid state is given by

$$
\begin{aligned}
P_{\mathrm{i} 1}= & \left(\varepsilon^{*} z^{\prime} / v^{*}\right) K s(\tilde{V}-1+s)^{-2} \\
& \times\left\{1-h-h \tilde{V}(\tilde{V}-1+s)^{-1}\left(\partial \ln K / \partial \ln V^{*}\right)^{-1}\right\}^{-1}
\end{aligned}
$$

where

$$
\begin{aligned}
K \equiv & -\varphi(0) / 2 \varepsilon^{*} \\
& =\left\{2.409\left(\sigma^{3} N / V^{*}\right)^{2}-1.0109\left(\sigma^{3} N / V^{*}\right)^{4}\right\} / 2
\end{aligned}
$$

and

$$
h \equiv \tilde{V}\left(\partial \tilde{V}^{*} / \partial T\right)_{p} /(\partial V / \partial T)_{p}
$$

for the general case in which $V^{*}$ and $\tilde{V}$ are both variable with temperature and pressure. For the model in which the cell volume is not variable, we have the alternative equation represented by ${ }^{1}$

$$
P_{\mathrm{i} 1}=\left(\varepsilon^{*} z^{\prime} / v^{*}\right) K s(\tilde{V}-1+s)^{-2}
$$

In the former case, we obtain

$$
\begin{aligned}
& \left(\frac{P_{\mathrm{ig}}}{P_{\mathrm{i} 1}}\right)_{T=T_{\mathrm{g}}} \\
& =\frac{2\left(1-h_{\mathrm{g}}\right)\left(\tilde{V}_{\mathrm{g}}-1+s\right)\left\{2.409-2.0218\left(\sigma^{3} N / V_{\mathrm{g}}{ }^{*}\right)^{2}\right\}}{\tilde{V}_{\mathrm{g}}\left\{2.0409-1.0109\left(\sigma^{3} N / V_{\mathrm{g}}{ }^{*}\right)^{2}\right\}} \\
& \quad+h_{\mathrm{g}}
\end{aligned}
$$

from eq 11 and 12, and

$$
\begin{aligned}
& \left(\frac{P_{\text {ig }}}{P_{\text {i1 }}}\right)_{T=T_{\mathrm{g}}} \\
& =\frac{2\left(\widetilde{V}_{\mathrm{g}}-1+s\right)\left\{2.409-2.0218\left(\sigma^{3} N / V_{\mathrm{g}}{ }^{*}\right)^{2}\right\}}{\widetilde{V}_{\mathrm{g}}\left\{2.409-1.0109\left(\sigma^{3} N / V_{\mathrm{g}}{ }^{*}\right)^{2}\right\}}
\end{aligned}
$$

from eq 11 and 15. These equations represent the change in internal pressure at glass transition temperature $T_{\mathrm{g}}$.

We can also estimate the change in internal pressure at $T_{\mathrm{g}}$ by another method which was used by Allen, et al. ${ }^{5}$ Since the configuration of chain molecules in the system may be frozen at $T_{\mathrm{g}}$, the decrease in internal pressure at $T_{\mathrm{g}}$ may be attributed to the disappearance of the pressure effect arising from the entropy $S^{\mathrm{c}}$ as- 
sociated with the combinatory factor, i.e., the arrangement of chain molecules on the lattice. The decrease in internal pressure, $\Delta P_{\mathrm{i}}$, is then given by eq 2 as

$$
\begin{aligned}
\Delta P_{\mathrm{i}}= & P_{\mathrm{i} 1}-P_{\mathrm{ig}}=T\left(\partial S^{\mathrm{c}} / \partial V\right)_{T}=R T\left(\partial \ln \sum_{\lambda} g_{\lambda} / \partial V\right)_{T} \\
= & \left\{\ln \left(1-\tilde{V}_{\mathrm{g}}{ }^{-1}\right)^{-1}-\widetilde{V}_{\mathrm{g}}{ }^{-1}+1 / r\right\} \widetilde{V}_{\mathrm{g}} \\
& \left.\times\left\{1-\partial \ln \tilde{V}^{*} / \partial \ln V\right)_{T}\right\} R T_{\mathrm{g}} / V_{\mathrm{g}}
\end{aligned}
$$

When $\left(\partial \ln V^{*} / \partial \ln V\right)_{T}=0$, eq 18 becomes the equation derived by Allen, et al. ${ }^{5}$ where they assumed the cell volume was constant, being independent of temperature and pressure.

\section{DISCUSSION AND COMPARISON WITH EXPERIMENTAL OBSERVATIONS}

Volume-Temperature Relation at Zero Pressure

The equation of state represented by eq 7 is identical with one of the two equations (eq 21 in ref 1) which determine the equation of state in the liquid state, except that the hole fraction (or $\tilde{V}$ ) is invariable in eq 7 (see APPENDIX). As also pointed out in the previous paper, ${ }^{1}$ the relation of $V^{*} \sigma^{3} N-\tilde{T}-\widetilde{P}$, expressed by eq 7 , is insensitive to the variations of $\tilde{V}$ and $s$ at pressure in the vicinity of atmospheric pressure. Accordingly, (1) the change in cell volume $V^{*}$ with temperature and pressure is almost the same in both the liquid and glassy states although the hole fraction (or $\tilde{V}$ ) is variable in the former state and invariable in the latter state; (2) the $P-V^{*}-T$ relation is almost identical with the $P-V-T$ relation in Simha's theory ${ }^{6}$ which is derived from eq 7 with $\widetilde{V}_{\mathrm{g}}=1$, and the volume $V$ estimated from eq 7 is the sum of the cell volume showing such behavior and the volume of frozen holes; and (3) the $\widetilde{P}-\tilde{T}$ $V^{*} / \sigma^{3} N$ relation is approximately a universal function independent of substances.

The relations of $V / \sigma^{3} N-\tilde{T}$ and $V^{*} / \sigma^{3} N-\tilde{T}$ at $P=0$ calculated from eq 7 are compared with some experimental results in Figure 1. The experimental volume-temperature relations were transformed into reduced forms with the molecular parameters obtained previously. ${ }^{1}$ As already pointed out, the $V / \sigma^{3} N-\tilde{T}$ relation depends on $\tilde{V}_{\mathrm{g}}$ or $V_{\mathrm{g}} / \sigma^{3} N$. In the calculation of $V / \sigma^{3} N-\tilde{T}$ curves for comparison with experimental results we were obliged to use the observed value of glass transition temperature (or

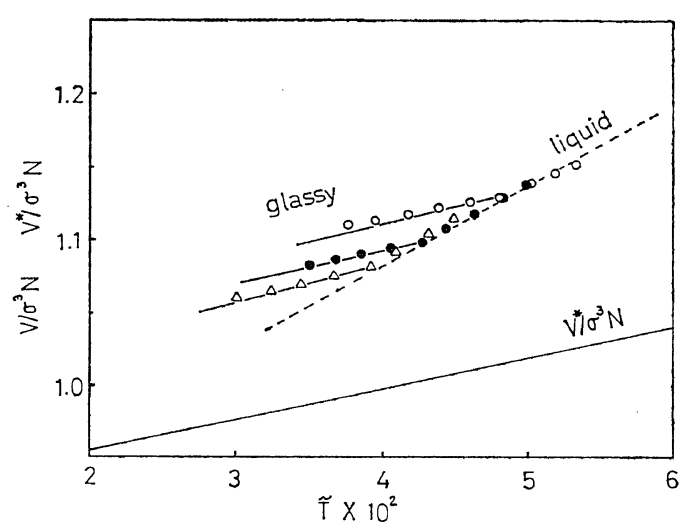

Figure 1. Reduced volume and reduced cell volume $v s$. reduced temperature under atmospheric pressure: - calculated; experimental, $\bigcirc$, poly(vinyl acetate $)^{5} ; 0$, polystyrene ${ }^{16} ; \triangle$, poly (methyl methacrylate) ${ }^{19}$ ( $c f$. Table II).

volume) to determine $\tilde{V}_{\text {g }}$, since we could not determine the glass transition temperature by the theory.

The calculated curves agree with the observed ones fairly well. This is in accordance with the well known fact that the lattice theory is valid for the solid state rather than for the liquid state.

Cohesive Energy Density and Internal Pressure

In Figure 2, we show the calculated curves for internal pressure and cohesive energy density in both the glassy and liquid states, comparing them with some experimental results, to indicate their characteristic behavior in the two states. As can be seen in Figure 2, internal pressure decreases sharply or discontinuously at $T_{g}$ as the substance is cooled from the liquid state to the glassy state. On the other hand, cohesive energy density does not show such change and increases continuously, as the volume decreases, with somewhat different gradients in the liquid and glassy states. Thus in the glassy state internal pressure is much less than cohesive energy density, whereas in the liquid state they are nearly equal to each other. Therefore it must be noted that in the glassy state internal pressure is no longer a direct measure of cohesive energy density. One more characteristic difference in internal pressure between the glassy and liquid states is that in the glassy state it increases as the volume increases, whereas in the liquid state 


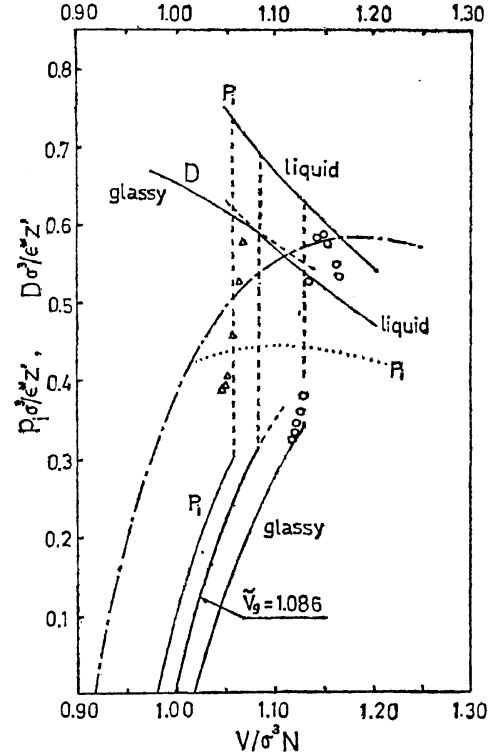

Figure 2. Reduced internal pressure and reduced cohesive energy density $v s$. reduced volume under atmospheric pressure in the liquid and glassy states: —- - --- --- and -----, calculated value; the solid and dotted lines in the liquid state are calculated by eq 15 with $r=\infty$ and eq 12 with $r=\infty$, respectively. - - indicates reduced internal pressure of the closest packing solid (eq 11 with $\widetilde{V}_{\mathrm{g}}=0$ ), which is identical with Simha's theory. ${ }^{6}$ Experimental: $\bigcirc$, poly(vinyl acetate) ${ }^{5}$ (cf. Table II, $\left.\varepsilon^{*} z^{\prime} / \sigma^{3}=178 \mathrm{cal} / \mathrm{cc}^{1}\right)$ and $\triangle$, poly (ethyl acrylate $)^{5}$ $\left(V_{\mathrm{g}}=0.855 \mathrm{cc} / \mathrm{g}, V_{\mathrm{g}} / \sigma^{3} N=1.058, \varepsilon^{*} z^{\prime} / \sigma^{3}=165 \mathrm{cal} / \mathrm{cc}^{1}\right)$.

it decreases, as is well known, approximately proportional to $V^{-2}$. It can be seen from Figure 2 that the above characteristic behavior of internal pressure can, at least qualitatively, be interpreted by the present hole model. Qualitatively speaking with the hole model, because of the change in the number of holes, internal pressure decreases as the volume increases in the liquid state, whereas since only the cell volume is changeable in the glassy state, it increases as the volume increases and is smaller in magnitude than that in the closest packing state (indicated by a chain line in Figure 1) due to the presence of frozen holes.

We now consider the internal pressure of the densified glass having a smaller value of $\tilde{V}$ which is obtained by glass-formation under elevated pressure. The slowly cooled glass has also less hole fraction and should show the same behavior in internal pressure. According to the results (1) in the preceding section, the $P-V^{*}-$ $T$ relation of the densified glass should be almost identical with that of nondensified glass, i.e.,

$$
\begin{gathered}
V(T, P) / V_{\mathrm{s}}(T, P)=\tilde{V}_{\mathrm{g}} / \tilde{V}_{\mathrm{gs}}=d \\
\text { (independent of } T \text { and } P \text { ) }
\end{gathered}
$$

where $V$ and $V_{\mathrm{s}}$ are the volume of the glasses formed under elevated and atmospheric pressures, respectively. Therefore, the thermal expansion coefficient $\alpha$ and compressibility $\beta$, hence internal pressure $\boldsymbol{P}_{\mathrm{i}}(\equiv \alpha / \beta-\boldsymbol{P})$, are almost the same for any glasses formed under different condition. However, it may not hold exactly. The ratio of internal pressure of the densified glass, $P_{\mathrm{i}}$, to that of nondensified one, $P_{\mathrm{is}}$, is derived from eq 11 as

$$
\begin{aligned}
\left(\boldsymbol{P}_{\mathrm{i}} / P_{\mathrm{is}}\right)_{T}= & =\tilde{V}_{\mathrm{gs}}\left(\tilde{V}_{\mathrm{gs}}-1+s\right) / \tilde{V}_{\mathrm{g}}\left(\tilde{V}_{\mathrm{g}}-1+s\right) \\
& \cong\left(\tilde{V}_{\mathrm{gs}} / \tilde{V}_{\mathrm{g}}\right)^{2}=d^{-2}
\end{aligned}
$$

if the variation of $V^{*} / \sigma^{3} N$ with $\tilde{V}_{\mathrm{g}}$ in the expression of eq 11 is assumed to be negligible. The values of the internal pressures $P_{\mathrm{i}}$ and $P_{\text {is }}$ compared at the same temperature and atmospheric pressure are very close but not equal. On the other hand, the ratio $\boldsymbol{P}_{\mathrm{i}} / \boldsymbol{P}_{\text {is }}$ compared at the same volume is expressed, from eq 11 , by

$$
\begin{aligned}
& \left(P_{\mathrm{i}} / P_{\mathrm{is}}\right)_{V} \\
& =\frac{\widetilde{V}_{\mathrm{g}}{ }^{2}\left(\tilde{V}_{\mathrm{gs}}-1+s\right)\left(2.409-2.0218 \tilde{V}_{\mathrm{g}}^{2} \tilde{V}^{\prime-2}\right)}{\tilde{V}_{\mathrm{gs}}^{2}\left(\tilde{V}_{\mathrm{g}}-1+s\right)\left(2.409-2.0218 \widetilde{V}_{\mathrm{gs}}^{2} \widetilde{V}^{\prime-2}\right)}
\end{aligned}
$$

Rearranging eq 21 , we have

$$
\begin{aligned}
& \left(P_{\mathrm{i} /} / P_{\mathrm{is}}\right)_{V} \\
& \quad=d\left[1+\left(1-d^{2}\right)\left(\sigma^{3} N / V^{*}\right)^{2} /\left\{1.192-\left(\sigma^{3} N / V^{*}\right)^{2}\right\}\right] \\
& \quad=d\left\{1-0.839\left(\sigma^{2} N / V^{*}\right)^{2} d^{2}\right\} /\left\{1-0.839\left(\sigma^{3} N / V^{*}\right)^{2}\right\}
\end{aligned}
$$

The value of $\left(P_{\mathrm{i}} / P_{\mathrm{is}}\right)_{T}$ calculated by eq 20 is 1.04 for $d=0.98$ and 1.11 for $d=0.95$, whereas that of $\left(\boldsymbol{P}_{\mathrm{i}} / \boldsymbol{P}_{\mathrm{is}}\right)_{V}$ calculated by eq 21 is 1.24 for $d=0.98$ and 1.44 for $d=0.95$, when $V^{*}=\sigma^{3} N$. The internal pressure compared at the same volume should vary more greatly with the magnitude of densification $d$ than that compared at the same temperature, whereas within a glass internal pressure is a function of volume only 
according to eq 11 and is invariable under constant volume. Experimental observations to examine eq 20 and 22, however, seem not to have been made yet.

Change in Internal Pressure at $T_{g}$

According to eq 16 and 17, if the principle of corresponding states holds, the ratio $\boldsymbol{P}_{\text {ig }} / \boldsymbol{P}_{\text {i1 }}$ should be dependent only on $\tilde{V}_{\text {g }}$, because the values of $h_{\mathrm{g}}$ and $\sigma^{3} N / V_{\mathrm{g}}{ }^{*}$ are determined by the value of $\tilde{V}_{\mathrm{g}}$. Since the value of $\tilde{V}_{\mathrm{g}}$ is, as will be shown later, almost the same for many polymers and furthermore the dependence of the above ratio on $\tilde{V}_{\mathrm{g}}$ is mild, the ratio expressed by eq 16 or 17 may have almost the same value for different polymers. (The $\tilde{V}_{\mathrm{g}}$ values for PVAc and PMMA in Figure 2 are exceptionally high and low respectively). If we take $V_{\mathrm{g}} / V_{0}=1.181$, the average value for some polymers listed in Table II, and use the values of $h_{\mathrm{g}}$ and $\sigma^{3} N / V_{\mathrm{g}}{ }^{*}$ obtained from Figure 1, the value of $\boldsymbol{P}_{\mathrm{ig}} / \boldsymbol{P}_{\mathrm{i} 1}$ at glass transition temperature is evaluated to be 0.707 from eq 16 and 0.451 from eq 17. The experimental values are calculated from published data to be shown in Table I. As already found in the previous section, the theoretical predictions for $\boldsymbol{P}_{\mathrm{ig}} / \boldsymbol{P}_{\mathrm{i} 1}$ give again satisfactory results semiquantitatively.

We now evaluate $\Delta P_{\mathrm{i}}$ with eq 18 , taking poly(vinyl acetate) (PVAc) as an example. It we put $\tilde{V}_{\mathrm{g}}=1.112, \quad r=\infty, \quad\left(\partial \ln V^{*} / \partial \ln V\right)_{T}=$ $\beta_{\mathrm{g}} / \beta_{1}=0.603$ (see eq A-11 in APPENDIX), $V_{\mathrm{g}}=$ $0.836 \times 86 / 2, \mathrm{cc} / \mathrm{mol}$ and $T_{\mathrm{g}}=294^{\circ} \mathrm{K}$ for PVAc, ${ }^{5}$ taking a half of repeating unit as a rotational unit of chain backbones, the value of $\Delta P_{\mathrm{i}}$ given by eq 18 is $10.0 \mathrm{cal} / \mathrm{cc}$. This value is much smaller than the observed value ${ }^{5}$ for PVAc, 40 $\mathrm{cal} / \mathrm{cc}$. Allen, et al., ${ }^{5}$ were led to the similar

Table I. Change in internal pressure at glass transition

\begin{tabular}{lcrc}
\hline \multicolumn{1}{c}{ Polymer } & $\begin{array}{c}\boldsymbol{P}_{\mathrm{ig}}, \\
\mathrm{cal} / \mathrm{cc}\end{array}$ & $\begin{array}{c}\boldsymbol{P}_{\mathrm{i} 1,}, \\
\mathrm{cal} / \mathrm{cc}\end{array}$ & $\boldsymbol{P}_{\mathrm{ig}} / \boldsymbol{P}_{\mathrm{i} 1}$ \\
\hline Polystyrene $^{\mathrm{a}}$ & 69 & 102 & 0.68 \\
Poly(methyl methacrylate) $^{\mathrm{a}}$ & 63 & 102 & 0.62 \\
Poly(vinyl chloride) $^{\mathrm{a}, \mathrm{b}, \mathrm{c}}$ & 46 & 83 & 0.55 \\
Poly(vinyl acetate) $^{\mathrm{d}}$ & 70 & 114 & 0.61 \\
& 70 & 111 & 0.63 \\
\hline
\end{tabular}

a ref $8 .{ }^{b}$ ref $9 . \quad$ ref 10. d ref 5 . conclusion but they obtained a much larger value, $22 \mathrm{cal} / \mathrm{cc}$, than ours in spite of taking a repeating unit as a rotational unit, because they calculated it by assuming that the volume of the cell was constant and putting $\tilde{V}_{\mathrm{g}}-1=0.025$ which is usually used as the free volume fraction at $T_{\mathrm{g}}$.

The former estimation with eq 16 and 17 was made by evaluating the change in the volume dependence of internal energy associated with intersegmental interactions, whereas the latter was derived from the volume dependence of the entropy associated with the combinatory factor. ${ }^{*}$ The disagreement between theoretical and experimental results in the latter estimation may arise from the difficulty in the evaluation of configurational entropy or the "communal entropy" of the liquid, which it is hardly possible to estimate by the usual lattice theory, as suggested in the previous paper. ${ }^{1}$

Reduced Volume and Reduced Temperature at $T_{g}$

Recently several investigators ${ }^{11-13}$ have shown that glass transition occurs at constant $\tilde{V}$ or $\tilde{T}$, and that the free volume fraction at $T_{\mathrm{g}},(1-$ $\left.V_{0} / V_{\mathrm{g}}\right)$, is $0.16-0.18^{11,12}$ or $0.13,{ }^{13,14}$ which is much less than the free volume fraction (about

* If, in the calculations of eq 16 and 18, all of the quantities involved in the equations are evaluated with the equation of state derived from the partition function eq 1 of the present theory, eq 16 and 18 must give the same result for the change in internal pressure at $T_{g}$ (though it is expressed by the ratio of $P_{i g}$ to $P_{i l}$ in eq 16 whereas by the difference $\Delta P_{i}$ in eq 18), because both of them are based on the same partition function of eq 1 . In the present numerical calculations of eq 16 and 18 , however, the quantities such as $h_{g}, V_{g}, \widetilde{V}_{g}$, and $\left(\partial \ln V^{*} / \partial \ln V\right)_{T}$ were evaluated with the aid of the values and relations obtained experimentally, and hence eq 16 and 18 gave different results. The use of the experimental values and relations may imply such correction for the partition function that the equations of state derived reproduces the experimental one. In the evaluation of eq 16 , the correction is considered to be that for the configurational entropy or the communal entropy, as pointed out in the preceding paper, ${ }^{1}$ whereas in the calculation of eq 18 it may be that for the terms of partition function excluding the combinatory factor, or the configurational entropy. 


\section{A Hole Theory of Polymer Liquids- and Glasses}

Table II. Some characterictic values concerning glass transition

\begin{tabular}{|c|c|c|c|c|c|c|c|}
\hline Polymer & $T_{\mathrm{g}},{ }^{\circ} \mathrm{K}$ & $V_{\mathrm{g}}, \mathrm{cc} / \mathrm{g}$ & $V_{0}^{+}, \mathrm{cc} / \mathrm{g}$ & $V_{\mathrm{g}} / V_{0}$ & $\begin{array}{l}E_{0} V_{0}^{+} / c \\
\mathrm{kcal} / \mathrm{mol}\end{array}$ & $\begin{array}{c}T_{\mathrm{g}} c / E_{0} V_{0} \\
\mathrm{deg} \mathrm{mol} / \mathrm{kcal}\end{array}$ & $\tilde{T}_{\mathrm{g}} \times 10^{2}$ \\
\hline Polystyrene ${ }^{a}$ & 371 & 0.968 & 0.808 & 1.198 & $12.3_{7}$ & 30.0 & 4.28 \\
\hline Poly $(\alpha \text {-methyl styrene })^{b}$ & 438 & 0.952 & 0.830 & 1.148 & $17.8_{2}$ & 24.6 & 3.51 \\
\hline Poly(vinyl acetate $)^{c}$ & 294 & 0.836 & 0.678 & 1.232 & $8.7_{7}$ & 33.5 & 4.78 \\
\hline Poly(ethyl acrylate) $)^{d, e}$ & 251 & 0.868 & 0.723 & 1.201 & $8.7_{1}$ & 29.2 & 4.11 \\
\hline Poly(methyl methacrylate) ${ }^{f}$ & 378 & 0.870 & 0.740 & 1.176 & $13.7_{8}$ & 27.4 & 3.90 \\
\hline Poly(ethyl methacrylate) ${ }^{\mathrm{f}}$ & 338 & 0.900 & 0.772 & 1.167 & $12.9_{6}$ & 26.0 & 2.78 \\
\hline $\operatorname{Poly}(n \text {-propyl methacrylate })^{f}$ & 308 & $0.931_{5}$ & 0.797 & 1.168 & $11.7_{4}$ & 26.3 & 3.74 \\
\hline $\operatorname{Poly}(n \text {-butyl methacrylate })^{\mathrm{f}}$ & 293 & $0.947_{5}$ & 0.817 & 1.159 & $11.4_{0}$ & 25.7 & 3.67 \\
\hline Average & & & & 1.181 & & $(27.8)$ & 3.96 \\
\hline
\end{tabular}

a ref $16, \quad$ b ref $17, \quad$ c ref 5, d $\operatorname{ref} 7,{ }^{\circ}$ ref $18, \quad$ \& ref $19,+\operatorname{ref} 1$.

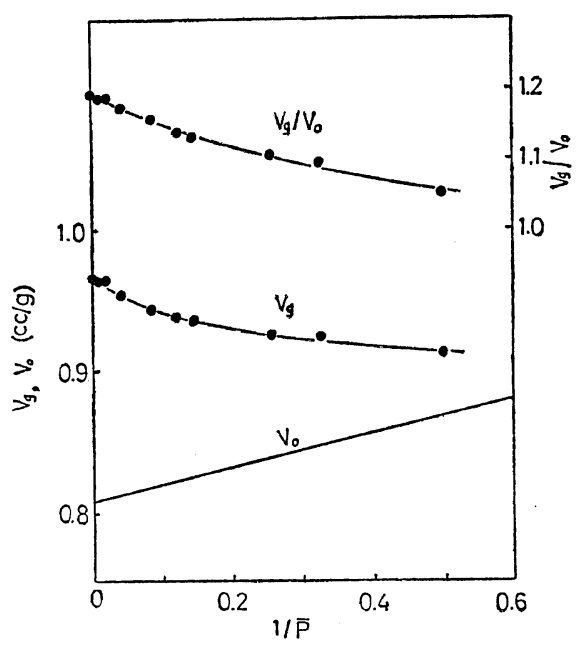

Figure 3. Dependences of $V_{0}, V_{\mathrm{g}}$ and $V_{\mathrm{g}} / V_{0}$ on the degree of polymerization $\bar{P}$ for polystyrene. ${ }^{16}$

$0.025)^{15}$ estimated from viscoelastic data with the Doolittle equation, where $V_{0}$ is the volume of hypothetical liquid at $0^{\circ} \mathrm{K}$. The constancy of $\tilde{V}_{\mathrm{g}}$ or $V_{\mathrm{g}} / V_{0}$ is equivalent to that of $\tilde{T}_{\mathrm{g}}$ according the principle of corresponding states. The values of $V_{\mathrm{g}} / V_{0}$ and $\tilde{T}_{\mathrm{g}}$ calculated with molecular parameters obtained in our analysis ${ }^{1}$ are tabulated in Table II, where $V_{0}$ represents the cell volume at $0^{\circ} \mathrm{K}$ and is equal to $0.916 \sigma^{3} \mathrm{~N}$ in the present theory. ${ }^{1}$ The free volume fraction calculated from the average value of $V_{\mathrm{g}} / V_{0}$ is 0.153 , although the constancy of $V_{\mathrm{g}} / V_{0}$ or $\tilde{T}_{\mathrm{g}}$ is not very good. Examining the constancy of $V_{\mathrm{g}} / V_{0}$ for polystyrenes of various degrees of polymerization $\bar{P}$ with the data of Ueberreiter and Kanig, ${ }^{16}$ we obtained the results shown in Figure 3 . Here the values of $V_{0}$ were estimated by the equation $V_{0}=(84 \bar{P}+14) /(104 \bar{P}+2) \mathrm{cc} / \mathrm{g}$, where $V_{0}$ of $2[\mathrm{H}]$ of the chain ends was evaluated to be $14 \mathrm{cc} / \mathrm{mol}$, according to the estimate of Miller. ${ }^{11}$ The value of $V_{\mathrm{g}} / V_{0}$ depends on the degree of polymerization and increases up to the value of the same magnitude as other polymers with increasing $\bar{P}$. Miller $^{20}$ also showed a similar fact for $n$-alkanes and polyethylene in their glass transitions estimated by viscosity data. Namely he showed that the free volume fraction $f_{\mathrm{g}}$ at $T_{\mathrm{g}}$ decreases with increasing chain length, i.e., $f_{\mathrm{g}}\left(=1-V_{0} / V_{\mathrm{g}}\right)$ varies from 0.093 for $\left(-\mathrm{CH}_{2}-\right)_{\infty}$ to 0.050 for $n-\mathrm{C}_{5} \mathrm{H}_{12}$.

The fact that the constancy of $V_{\mathrm{g}} / V_{0}$ is not well satisfied may be attributed to intrasegmental interactions. At the present stage, however, this constancy may be considered to be a rough measure of the glass transition temperature in polymers.

Provided that the constancy of $V_{\mathrm{g}} / V_{0}$, hence that of $\tilde{T}_{\mathrm{g}}$, is a valid assumption, then we obtain from the definition of the reduced temperature

$$
T_{\mathrm{g}}=(0.718 R)^{-1} \tilde{T}_{\mathrm{g}} E_{0} V_{0} / c=\text { constant } \cdot E_{0} V_{0} / c
$$

where $E_{0}=0.718 \varepsilon^{*} z^{\prime} N / V_{0}$ is the cohesive energy density $D$ of hypothetical liquid at $0^{\circ} \mathrm{K}$ (i.e., at $\left.V=V^{*}=V_{0}\right)$. The eq 23 indicates that $T_{\mathrm{g}}$ is proportional not to $D$ itself, which is the cohesive energy per unit volume, but to $D V_{0} / c$, which is the cohesive energy per molar volume of a freely movable segment having three ex- 


\section{T. Nose}

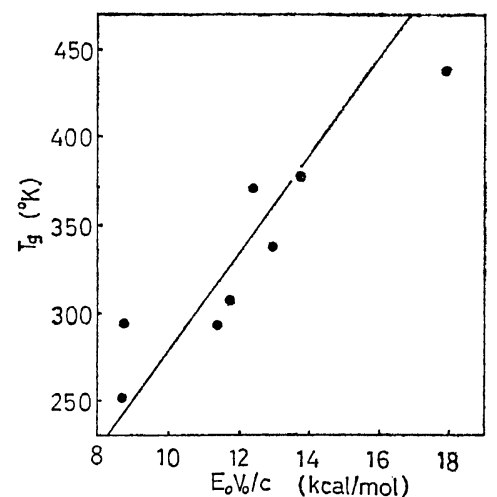

Figure 4. Relation of glass transition temperature to cohesive energy per freely movable segment: - eq 23 with $\widetilde{T}_{\mathrm{g}}=0.0396$; experimentals of P $\alpha$ MSt, PMMA, PEMA, P- $n$-PMA, PVAc, P- $n$ BMA, and PEA from higher $T_{\mathrm{g}}$ (data used are listed in Table II).

ternal degrees of freedom. The values of $T_{\mathrm{g}} /\left(E_{0} V_{0} / c\right)$ of the present analysis are shown in Table II. From Eq 23 with $\tilde{T}_{\mathrm{g}}=0.0396$, the average value in Table II, the proportional constant of eq 23 is obtained as $27.8 \mathrm{deg} \mathrm{mol} / \mathrm{kcal}$. The $T_{\mathrm{g}} v s . E_{0} V_{0} / c$ relations for the data in Table II are shown in Figure 4. This relation represented by eq 23 appears to be satisfied better than the linear relation ${ }^{21}$ between $T_{\mathrm{g}}$ and $D$.

From a consideration concerning $T_{\mathrm{g}}$ similar to the above, Turnbull and Cohen ${ }^{22}$ presented the relation $T_{\mathrm{g}}=0.023 h_{\mathrm{v}} / R$ for simple lowmolecular-weight substances, where $h_{\mathrm{v}}$ is heat of vaporization. This results means that the value of $\tilde{T}_{\mathrm{g}}$ in the present analysis should be $0.718 \times 0.023=0.0165$ from eq 23 with $h_{\mathrm{v}}=E_{0} V_{0}$ and $c=1$. This value is too much smaller than the value of 0.0396 obtained here for polymers. This is however consistent with the fact that the value of $V_{\mathrm{g}} / V_{0}$ or the free volume fraction at $T_{\mathrm{g}}$ decreases with increasing the chain length in polystyrene and $n$-alkane, as already mentioned. It is noteworthy that, being estimated by the extrapolation of the $V / \sigma^{3} N-\tilde{T}$ curve in the liquid state obtained previously ${ }^{1}$ (see Figure 1 ), the value of $V_{\mathrm{g}} / V_{0}$ corresponding to $\widetilde{T}_{\mathrm{g}}=$ 0.0165 is 1.04 , which is almost equal to the values of $V_{\mathrm{g}} / V_{0}$ for the lowest-molecular-weight oligostyrene, the dimer, shown in Figure 3 and that for $n-\mathrm{C}_{5} \mathrm{H}_{12}$, estimated by Miller. ${ }^{20}$
Acknowledgements. The author would like to thank Prof. T. Hata of the Tokyo Institute of Technology for encouraging discussions and much helpful advice.

\section{APPENDIX}

The free energy $F$ of a system is regarded as a function of $V, V^{*}$ and $T$ :

$$
F=F\left(\tilde{V}, V^{*}, T\right)
$$

Two of these three variables $\left(\tilde{V}, V^{*}\right.$, and $\left.T\right)$ are independent in the liquid state and the cell volume $V^{*}$, for example, is determined by

$$
\left(\partial F / \partial V^{*}\right)_{V, T}=0
$$

so as to minimize $F$, just as assumed in the previous paper. ${ }^{1}\left(A \equiv\left(\partial F / \partial V^{*}\right)_{V, T}\right.$ is called the "affinity" for $V^{*}$ and is zero in an equilibrium state.) From eq A-1, A-2, and a thermodynamic equation

$$
P=-(\partial F / \partial V)_{T}
$$

we have

$$
\left(\partial F / \partial V^{*}\right)_{V, T}=\left(\partial F / \partial V^{*}\right)_{\tilde{V}, T}-(\partial F / \partial \tilde{V})_{V^{*}, T} \tilde{V} / V^{*}=0
$$

and

$$
\begin{aligned}
P= & -(\partial F / \partial \tilde{V})_{V^{*} T}(\partial \tilde{V} / \partial V)_{T}-\left(\partial F / \partial V^{*}\right)_{\tilde{V}, T}\left(\partial V^{*} / \partial V\right)_{T} \\
= & -(\partial F / \partial \tilde{V})_{V^{*} T}\left\{1-\left(\partial V^{*} / \partial V\right)_{T} \tilde{V}\right\} / V^{*} \\
& -\left(\partial F / \partial V^{*}\right)_{\tilde{V}, T}\left(\partial V^{*} / \partial V\right)_{T}
\end{aligned}
$$

Combining eq A-4 and A-5, we obtain

$$
P \tilde{V}+\left(\partial F / \partial V^{*}\right)_{\tilde{V}, T}=0
$$

and

$$
P V^{*}+(\partial F / \partial \tilde{V})_{V^{*}, T}=0
$$

The equation of state in the liquid state is given by eq A-6 and A-7. On the other hand, the equation of state in the glassy state can be derived from $\boldsymbol{P}=-(\partial F / \partial V)_{T}$ with the condition $\tilde{V}=\tilde{V}_{\mathrm{g}}=$ constant to be expressed by

$$
P \tilde{V}_{\mathrm{g}}+\left(\partial F / \partial V^{*}\right)_{\tilde{V}, T}=0
$$

Accordingly it can be seen that the equation of state in the glassy state, (eq A-8), and eq A-6 expressed in the same form except that $\tilde{V}$ $\left(=V / V^{*}\right)$ may or may not be variable. Therefore if the variation of $\tilde{V}$ (or $\tilde{V}_{\mathrm{g}}$ ) does not 


\section{A Hole Theory of Polymer Liquids and Glasses}

greatly influence the $P-V^{*}-T$ relation in these expressions, as in the case of the model in the text, it follows that change in the cell volume with temperature and pressure is almost the same in both the glassy and liquid states. Thus we have

$$
\left(\partial \ln V^{*} / \partial T\right)_{T}{ }^{1} \cong\left(\partial \ln V^{*} / \partial T\right)_{P} \mathrm{~g}=(\partial \ln V / \partial T)_{P} \mathrm{~g}
$$

and

$$
\left(\partial \ln V^{*} / \partial P\right)_{T}{ }^{1} \cong\left(\partial \ln V^{*} / \partial P\right)_{T}{ }^{\mathrm{g}}=(\partial \ln V / \partial P)_{T}{ }^{\mathrm{g}}
$$

where the superscripts 1 and $g$ refer to the liquid and glassy states, respectively. The quantity $\left(\partial \ln V^{*} / \partial \ln V\right)_{T}$ at $T_{\mathrm{g}}$ in eq $18 \mathrm{can}$ be expressed by the ratio $\beta_{\mathrm{g}} / \beta_{1}$ of compressibilities in the glassy and liquid states, i.e.,

$$
\begin{aligned}
& \left(\partial \ln V^{*} / \partial \ln V\right)_{T} \\
& \quad=\left(\partial \ln V^{*} / \partial P\right)_{T}{ }^{1} /(\partial \ln V / \partial P)_{T}{ }^{1} \cong \beta_{\mathrm{g}} / \beta_{1}
\end{aligned}
$$

\section{LIST OF SYMBOLS}

$c$, one third of external degrees of freedom of a segment

$D$, cohesive energy density

$D_{\mathrm{g}}$, cohesive energy density in the glassy state

$d$, ratio of reduced volume $\tilde{V}$ of densified glass to nondensified glass, $\tilde{V}_{\mathrm{g}} / \tilde{V}_{\mathrm{gs}}$

$E_{0}$, cohesive energy density of hypothetical liquid at $0^{\circ} \mathrm{K}$, which is equal to 0.718 $\varepsilon^{*} z^{\prime} N / V_{0}$

$F$, Helmholtz free energy

$f_{\mathrm{g}}$, free volume fraction at glass transition temperature defined by $1-V_{0} / V_{\mathrm{g}}$

$\sum g_{\lambda}$, number of configurations of molecules arranged on lattice

$h$, ratio of thermal expansion coefficient of cell volume to volume defined by eq 14

$h_{\mathrm{g}}, h$ at glass transition temperature

$h_{\mathrm{v}}$, heat of vaporization

$J(T)$, partition function for intrasegmental degrees of freedom

$K$, function defined by eq 13

$k$, Boltzmann's constant

$M$, total number of lattice sites in the system

$N$, total number of segments in the system

$P$, pressure

$\widetilde{P}$, reduced pressure, $P v^{*} / \varepsilon^{*} z^{\prime}$
$\bar{P}$, degree of polymerization

$P_{\mathrm{i}}$, internal pressure, $(\partial U / \partial V)_{T}$

$P_{\text {ig }}$, internal pressure in the glassy state

$P_{i 1}$, internal pressure in the liquid state

$P_{\text {is }}$, internal pressure of the glass formed under atmospheric pressure

$\Delta \boldsymbol{P}_{\mathrm{i}}$, difference in internal pressure at glass transition temperature between the liquid and glassy states, $P_{\mathrm{i} 1}-P_{\mathrm{ig}}$ at $T_{\mathrm{g}}$

$q$, kinetic part of partition function associated with external degrees of freedom

$R$, gas constant

$r$, number of segments of a polymer chain

$S^{\mathrm{e}}$, entropy associated with combinatory factor

$s$, ratio of coordination number of a segment to that of a lattice site, $z^{\prime} / z$

$T$, absolute temperature

$\tilde{T}$, reduced temperature, $c k T / \varepsilon^{*} z^{\prime}$

$T_{\mathrm{g}}$, glass transition temperature

$\widetilde{T}_{\mathrm{g}}$, reduced temperature at glass transition temperature

$U$, internal energy

$V$, volume

$V^{*}$, volume of cells occupied by segments, $v^{*} N$

$\tilde{V}$, reduced volume defined by $\tilde{V}=V / V^{*}=$ $M / N$

$\tilde{V}^{\prime}$, reduced volume defined by $\tilde{V}^{\prime}=V / \sigma^{3} N$

$V_{\mathrm{g}}$, volume at glass transition temperature

$V_{\mathrm{g}}{ }^{*}, V^{*}$ at glass transition temperature

$\widetilde{V}_{\mathrm{g}}$, reduced volume $\tilde{V}$ at glass transition temperature

$V_{\mathrm{s}}$, volume of the glass formed under atmospheric pressure

$\tilde{V}_{\mathrm{gs}}$, reduced volume $\tilde{V}$ of the glass formed under atmospheric pressure

$V_{0}$, volume of hypothetical liquid at $0^{\circ} \mathrm{K}$, which is equal to $V^{*}$ at $0^{\circ} \mathrm{K}$

$v^{*}$, volume of a cell

$v_{\mathrm{f} 1}$, free volume given by eq 3

$v_{\text {fo }}$, free volume given by eq 4

$Z$, partition function

$Z_{\nabla}$, volume-dependent part of partition function

$z$, coordination number of a lattice site

$z^{\prime}$, coordination number of a segment

$\alpha$, thermal expansion coefficient

$\beta$, isothermal compressibility

$\beta_{3}$, isothermal compressibility in the glassy state 
$\beta_{1}$, isothermal compressibility in the liquid state

$\varepsilon^{*}$, potential energy at minimum of potential energy curve of Lennard-Jones 12-6 potential

$\sigma$, distance between segment centers at which potential energy is zero in LennardJones $12-6$ potential

$\varphi(0)$, potential energy of intersegmental interactions when the segments are at the centers of the cell as given by eq 5 .

\section{REFERENCES}

1. T. Nose, Polymer J., 2, 124 (1971).

2. I. Prigogine, "The Molecular theory of Solutions", Interscience publishers Inc., New York, N.Y., 1957.

3. P. J. Flory, R. A. Orwoll, and A. Vrij, $J$. Amer. Chem. Soc., 86, 3507 (1964).

4. P. J. Flory, "Principles of Polymer Chemistry", Cornell University Press, Ithaca, N.Y., 1953.

5. G. Allen, D. Sims, and G. J. Wilson, Polymer, 2, 375 (1961).

6. V.S. Nanda and R. Simha, J. Phys. Chem., 68, 3158 (1964).

7. G. Allen, G. Gee, D. Mangaraj, D. Sims, and G. J. Wilson, Polymer, 1, 467 (1960).

8. K. H. Hellwege, W. Knappe, and P. Lehmann,
Kolloid-Z., 183, 110 (1962).

9. G. Gee, Polymer, 7, 177 (1966).

10. P. Heydemann and H. D. Guicking Kolloid-Z., 193, 16 (1963).

11. A. A. Miller, J. Polym. Sci., Part A, 4, 415 (1966).

12. R. Simha and R. F. Boyer, J. Chem. Phys., 37, 1003 (1962).

13. A. Bondi, J. Polym. Sci., Part A, 2, 3159 (1964).

14. R. N. Haward, H. Breuer, and G. Rehage, $J$. Polym. Sci., Part B, 4, 375 (1966).

15. See, for example, J. D. Ferry, "Viscoelastic Properties of Polymers", John Wiley and Sons. Inc., New York, N.Y., 1961, p. 201.

16. K. Ueberreiter and G. Kanig, J. Colloid Sci., 7, 569 (1952).

17. T. Yasuda and T. Hata, the 19th Annual Meeting of the Chemical Society of Japan, Yokohama, 1966; unpublished data; private communication.

18. R. H. Wiley and G. H. Braner, J. Polym. Sci., 3, 647 (1948).

19. S. S. Rogers and L. Mandelkern, J. Phys. Chem., 61, 945 (1957).

20. A. A. Miller, J. Polym. Sci., Part A, 6, 249 (1968).

21. R. Kawai, Kobunshi, (High Polymers, Japan), 6, 348 (1957).

22. D. Turnbull and M. H. Cohen, J. Chem. Phys., 34, 120 (1961). 